\title{
Cinzas de casca de arroz como fonte alternativa de silício para a síntese de zeólita beta
}

\author{
Heveline Enzweiler', Elisa Barbosa-Coutinho², Marcio Schwaab³ \\ 'Doutora em Engenharia Química - Universidade Federal de Santa Maria, Santa Maria, RS - Brasil \\ ${ }^{2}$ Doutor em Engenharia Química - Universidade Federal de Santa Maria, Santa Maria, RS - Brasil
}

\begin{abstract}
Resumo
Cinzas de casca de arroz são resíduos agroindustriais gerados em larga escala que podem acarretar problemas ambientais e riscos à saúde da população. Como solução para este problema, as cinzas podem ser utilizadas como fonte alternativa de sílica altamente reativa, com vasto campo de aplicação, como, por exemplo, na síntese de catalisadores de alto teor de silício, como as zeólitas. Neste trabalho, zeólita beta foi sintetizada usando sílica amorfa, extraída de cinzas de casca de arroz, e pseudoboemita. Utilizando esta fonte alternativa de silício foi possível a formação da estrutura zeolítica com excelentes propriedades catalíticas. A aplicação da zeólita beta na desidratação do isopropanol possibilitou a produção de propeno com alta seletividade a baixas temperaturas, mostrando que a utilização de sílica proveniente de cinzas de cascas de arroz tem grande potencial tecnológico e ambiental.
\end{abstract}

Palavras-chave: Zeólita beta; catalisador; cinzas de casca de arroz; isopropanol; propeno.

\begin{abstract}
Rice husk ash is an agroindustrial residue generated in large scale that can cause environmental problems and risks to people health. As a solution to this problem, this ash could be used as an alternative source of high reactive silica, with a wide field of applications, such as, for example, in the synthesis of catalysts with high silicon content. In this work, beta zeolite was synthesized using amorphous silica, extracted from rice husk ash, and pseudo-boehmite. Using this alternative source of silicon, it was possible to form the zeolitic structure with excellent catalytic properties. The application of the beta zeolite in the dehydration of isopropanol enables the production of propylene with high selectivity at low temperatures, showing that the use of silica from rice husk ash has a great technological and environmental potential.
\end{abstract}

Key-words: Beta zeolite; catalyst; rice husk ash; isopropanol; propylene. 


\section{INTRODUÇÃO}

Casca de arroz é um material residual abundante da produção agrícola e vem sendo utilizada com grande eficiência como fonte de energia através da queima. Contudo, a combustão gera o problema ambiental da disposição das cinzas (CHANDRASEKHAR et al., 2003). Em alguns lugares as cinzas de casca de arroz são consideradas como resíduo e são dispostas em aterros sanitários, causando poluição do ar e das águas (AN et al., 2011). Partículas de cinzas de casca de arroz carregadas pelo ar têm sido ligadas a doenças respiratórias em seres humanos (DEY et al., 2013).

Entretanto, a casca de arroz apresenta grande quantidade de sílica em sua composição (DELLA et al., 2002), podendo ser usada como fonte alternativa deste material. A queima da casca de arroz produz cinzas com teor de sílica entre 85 e 98\% (DEY et al., 2013; SARANGI et al., 2011). Quando a queima é realizada de forma controlada, é possível a obtenção de sílica altamente reativa (JAMES e RAO, 1986). A sílica de cinzas de casca de arroz pode ser usada como precursor para a síntese de diversos materiais, que podem ser muito úteis para aplicações industriais como catalisadores, tanto após um processo de extração e purificação (AN et al., 2010), como com o emprego direto da cinza. A utilização deste resíduo agroindustrial como fonte alternativa de sílica ainda reduz os custos de produção de catalisadores (JANG et al, 2009).

Catalisadores são fundamentais em uma sociedade industrial sustentável, pois aliam cuidados com o meio ambiente e viabilidade econômica. Com o uso de catalisadores têm-se processos com menor consumo energético, melhor uso dos recursos naturais, redução de subprodutos e eliminação de efluentes com potencial contaminante. Dentre os catalisadores heterogêneos, as zeólitas são o material mais utilizado, sendo amplamente aplicados em refinarias, petroquímicas e indústrias de química fina (MARTÍNEZ e CORMA, 2011).

Zeólitas são silicatos e aluminosilicatos cristalinos, com estrutura tridimensional formada por tetraedros de $\mathrm{SiO} 4$ e $\mathrm{AlO} 4$, ligados pelos átomos de oxigênio, contendo canais e cavidades de dimensões moleculares (CORMA, 2003). As zeólitas possuem grande área específica e alta capacidade de adsorção. São, ainda, considerados materiais com marcante estabilidade térmica e hidrotérmica (MARTÍNEZ e CORMA, 2011). Catalisadores zeolíticos possuem elevada acidez, sendo largamente aplicados em reações catalíticas heterogêneas que requerem sítios ácidos. $\mathrm{O}$ número e a força dos sítios ácidos podem ser controlados por métodos como a troca iônica, des/realuminação ou substituição isomórfica de átomos tetraédricos (O'CONNOR et al., 1996).

A zeólita beta foi sintetizada pela primeira vez por em 1967, sendo a sua estrutura determinada apenas em 1988 (ZAIKUET et al., 2001). É uma zeólita de poros grandes, sua estrutura favorece reações com compostos que possuem grande diâmetro cinético (TORRES e CARDOSO, 2008). Vem sendo usada como catalisador em várias reações, como alquilação do isobutano (CORMA et al., 1994), conversão de metanol a hidrocarbonetos (MIKKELSEN e KOLBOE, 1999), hidrocraqueamento e hidrodessulfurização (CAMBLOR et al., 1998).

A zeólita beta $(\beta)$ é tradicionalmente sintetizada em meio alcalino com íons de tretaetilamonio $(\mathrm{TEA}+)$ como direcionador de estrutura (KONG et al., 2009). As principais fontes de alumínio são aluminato de sódio, alumínio metálico, além da pseudoboemita (KONG et al., 2009), do sulfato de alumínio (SANTI et al., 2013), do nitrato de alumínio (VAN DER MYNSBRUGGE et al., 2012) e do caolin (DUAN et al., 2011). Como fontes de sílica encontram-se, principalmente, a sílica fumê, a sílica coloidal e o tetraetilortosilicato e, como alternativas, a sílica gel (WANG et al., 2004) e também a cinza da casca de arroz (PRASETYOKO et al., 2006).

O uso de cinza de casca de arroz já foi reportado na síntese de outras zeólitas, como mordenita (BAJPAIET et al., 1981), faujasitas (THUADAIJ e NUNTIYA, 2012; RAMLI et al., 1996), HZSM-48 (WANG et al., 1998) e ZSM-5 (DEY et al., 2013). PRASETYOKO e colaboradores (2006) avaliaram o uso direto de cinzas de casca de arroz na síntese de zeólita beta, estudando a transformação da cinza na estrutura da zeolítica durante o processo de cristalização.

Zeólitas, por suas características ácidas, são usadas como catalisadores em reações de desidratação, em especial, na desidratação de álcoois para a produção de olefinas (ZULFUGAROV et al., 1984; WEST et al., 2009). Dentre estas, o propeno é matéria-prima de grande interesse na indústria para a obtenção de uma infinidade de produtos químicos, principalmente, polímeros. A sua produção a partir de álcoois, neste caso o isopropanol, obtidos pela fermentação de açúcares, se mostra como uma alternativa renovável frente às fontes fósseis, como o petróleo (LARSEN et 
al., 1997), promovendo o seqüestro do $\mathrm{CO} 2$ do ambiente e fixando-o na forma de polímeros, minimizando os impactos causados pelo homem ao meio ambiente. A reação de conversão catalítica do isopropanol formando propeno ocorre em sítios ácidos (FERENS et al., 2012), em temperaturas entre $100^{\circ} \mathrm{C}$ e $350^{\circ} \mathrm{C}$, sendo as maiores conversões observadas a partir de $200^{\circ} \mathrm{C}$.

O objetivo deste trabalho foi a síntese de zeólita beta usando como fonte de sílica cinzas de casca de arroz, um resíduo agroindustrial, e a sua utilização na reação de desidratação do isopropanol para a produção de propeno verde. Assim, tem-se tanto o emprego de resíduo agroindustrial como matéria-prima para a síntese do catalisador, quanto um processo para produzir propeno a partir de uma fonte renovável.

\section{METODOLOGIA}

\section{I Extração de sílica da cinza de casca de arroz}

Para obtenção da sílica amorfa, inicialmente, a casca de arroz era lavada e seca para a remoção de contaminantes solúveis. A amostra era calcinada a cinza em mufla a $500{ }^{\circ} \mathrm{C}$, a $10^{\circ} \mathrm{C} /$ min, por 6 horas, para a remoção de compostos orgânicos. A seguir, a sílica era extraída da cinza como descrito por RAMLI e colaboradores (1996). Primeiramente, a cinza era adicionada à solução $2 \mathrm{M}$ de $\mathrm{NaOH}$ e a solução mantida sob agitação e aquecimento, $90^{\circ} \mathrm{C}$, por $2 \mathrm{~h}$. A solução de silicato de sódio, assim obtida, era filtrada, para a remoção de sólidos insolúveis, e a sílica precipitada pela adição lenta de ácido nítrico concentrado até $\mathrm{pH} 9$. O precipitado era filtrado e seco a $100^{\circ} \mathrm{C}$ por $24 \mathrm{~h}$.

\subsection{Síntese de zeólita beta}

A síntese da zeólita beta foi realizada conforme descrito por CARVALHO e CARDOSO (1997); trocando-se a fonte de sílica pela sílica amorfa extraída da cinza de casca de arroz. Empregou-se também pseudoboemita e hidróxido de tetraetilamônio como fonte de alumínio e direcionador de estrutura. A composição molar do gel de síntese é descrita por 3Na2O.12,5(TEA)2O. $\mathrm{A} 12 \mathrm{O} 3.50 \mathrm{SiO} 2.1000 \mathrm{H} 2 \mathrm{O} .6 \mathrm{HCl}$. A cristalização estática foi realizada sob pressão autógena a 140 ${ }^{\circ} \mathrm{C}$, por 48 horas. A zeólita na forma sódica foi recuperada pela a adição de nitrato de sódio a quente, seguida de centrifugação. A zeólita foi calcinada a $560{ }^{\circ} \mathrm{C}, 5^{\circ} \mathrm{C} / \mathrm{min}$, por oito horas para a remoção completa do direcionador orgânico.

A forma ácida da zeólita beta (H- $\beta)$ foi obtida a partir da forma sódica $(\mathrm{Na}-\beta)$ precursora através do processo de troca iônica. A Na- $\beta$ foi mantida em contato com solução $1 \mathrm{M}$ de nitrato de amônio, sob agitação por 4 horas à $70^{\circ} \mathrm{C}$, seguida de centrifugação e secagem a $80^{\circ} \mathrm{C}$ durante a noite. A amostra resultante foi novamente calcinada nas mesmas condições anteriores.

\subsection{Caracterização}

As amostras foram caracterizadas quanto a sua estrutura cristalina por difração de raios-X (Miniflex, Rigaku) com 30 kV, 10 mA e $\mathrm{Cu} \mathrm{K \alpha}$, como fonte de radiação, As propriedades texturais foram analisadas por fisissorção estática de nitrogênio a 77 K (ASAP 2020, Micromeritics).

\subsection{Atividade catalítica na desidratação de isopropanol a propeno}

As zeólitas foram empregadas como catalisadores na reação de desidratação de isopropanol a propeno. Os testes foram realizados em unidade de reação com reator tubular em "U" de quartzo, mantido em forno (Sanchis) com controle de temperatura (N1200, Novus), acoplado em linha com cromatógrafo a gás (CG 17-A, Shimadzu). As análises eram realizadas empregando-se uma coluna HP-Plot/Q de $30 \mathrm{~m}$, sob fluxo de $2,5 \mathrm{~mL} / \mathrm{min}$ de $\mathrm{N} 2$, com um programa de temperatura partindo de $100{ }^{\circ} \mathrm{C}$ por $8 \mathrm{~min}$ seguida de aquecimento até $185^{\circ} \mathrm{C}$ a $5^{\circ} \mathrm{C} / \mathrm{min}$, e outro aquecimento até 220 ${ }^{\circ} \mathrm{C}$ a $20^{\circ} \mathrm{C} / \mathrm{min}$, a temperatura final mantida por 5 min, com injeção automática por válvula com razão de split de 20, sendo o injetor mantido a 175 ${ }^{\circ} \mathrm{C}$, e o detector de ionização em chama mantido a $250^{\circ} \mathrm{C}$.

Primeiramente, $30 \mathrm{mg}$ de zeólita beta era tratada in situ a $200{ }^{\circ} \mathrm{C}$ sob fluxo de nitrogênio, 60 $\mathrm{mL} / \mathrm{min}$, por 2 horas. Em seguida, era alimentado isopropanol pela passagem de nitrogênio, $50 \mathrm{~mL} /$ min, em saturador a $15^{\circ} \mathrm{C}$. Variou-se a temperatura de reação de 100 até $225^{\circ} \mathrm{C}$, em intervalos de $25^{\circ} \mathrm{C}$.

\section{RESULTADOS E DISCUSSÃO}

Após dissolução das cinzas de casca de arroz em solução básica, seguida de precipitação em solução ácida foi possível obter a sílica com características de materiais amorfos, conforme difratograma de raios-X, Figura 1. O difratograma de raios-X apresenta um pico de difração entre 20 e $30^{\circ}$, característico de sílica. Como este pico de difração mostrou-se bastante alargado, pode-se supor que este material apresenta uma estrutura 


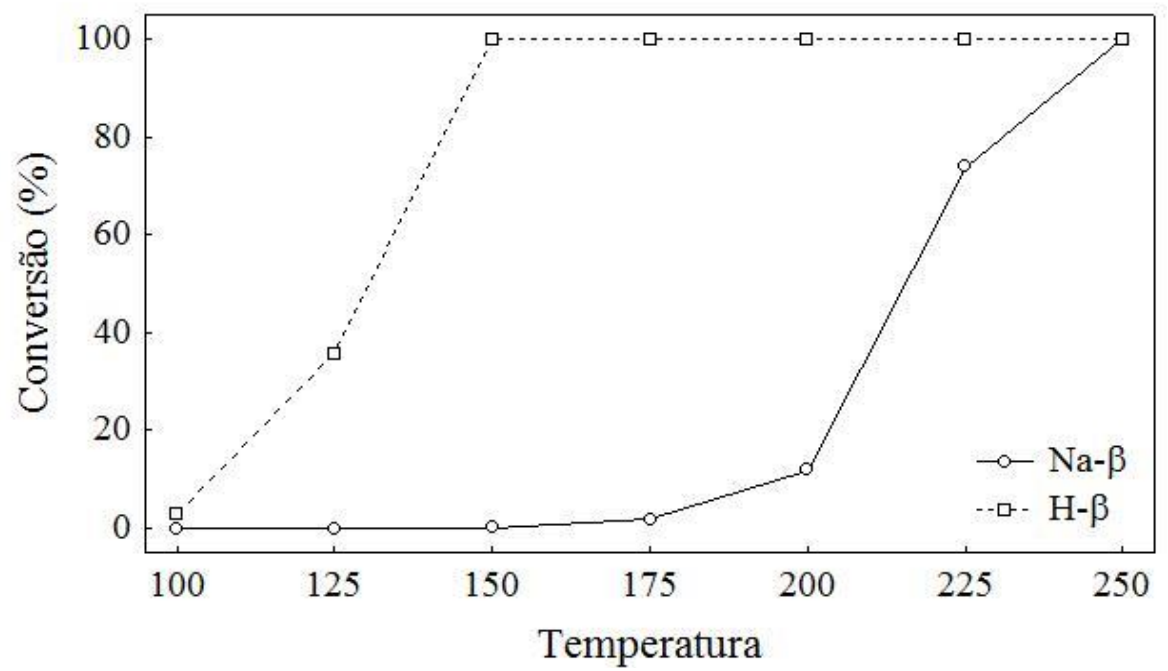

Figura 1 - Difratograma de raios-X de sílica extraída de cinzas de casca de arroz.

com baixa cristalinidade. Este resultado está em concordância com os obtidos por RAMLI e colaboradores (1996), AN e colaboradores (2010) e SARANGI e colaboradores (2011). Este material foi empregado como precursor para a síntese de zeólita beta.

A zeólita beta foi sintetizada empregando-se a sílica amorfa extraída da cinza da casca de arroz, sendo uma parte do material submetido à etapa de troca iônica para a obtenção da forma ácida. Suas estruturas cristalinas foram caracterizadas também por difração de raios-X, Figura 2. Pode-se observar que as zeólitas tanto na forma sódica quanto na forma ácida apresentam estrutura que corresponde à zeólita beta (CORMA et al., 2003; KUMAR et al., 2012; YIN et al., 2013) Os picos de difração em $2 \square$ de $7,8^{\circ}, 13,6^{\circ}, 14,5^{\circ} 21,5^{\circ} \mathrm{e}$ $22,5^{\circ}$ associados a estrutura da zeólita beta estão presentes em ambas as amostras, indicando que o processo de troca iônica não alterou a estrutura cristalina da zeólita.

As amostras também foram caracterizadas quanto a suas propriedades texturais pela adsorção/ dessorção de nitrogênio a $77 \mathrm{~K}$, cujas isotermas estão na Figura 3. O perfil das isotermas indica adsorção do gás em múltiplas camadas após a completa ocupação da camada monomolecular, que ocorre a pressões relativas próximas a 0,1 (ZHANG et al, 2013).

Observa-se zeólita beta na forma sódica adsorve uma maior quantidade de N2 por massa de amostra que esta zeólita na forma ácida nas mesmas pressões relativas, que fica evidenciado pelos seus valores de área específica e volume de poros. A zeólita beta na forma sódica apresentou elevada área específica, $506 \mathrm{~m}^{2} / \mathrm{g}$, volume de poros de $0,296 \mathrm{~cm}^{3} / \mathrm{g}$ e tamanho médio de poros de 23 $\AA$, indicando a presença de mesoporos e macro-

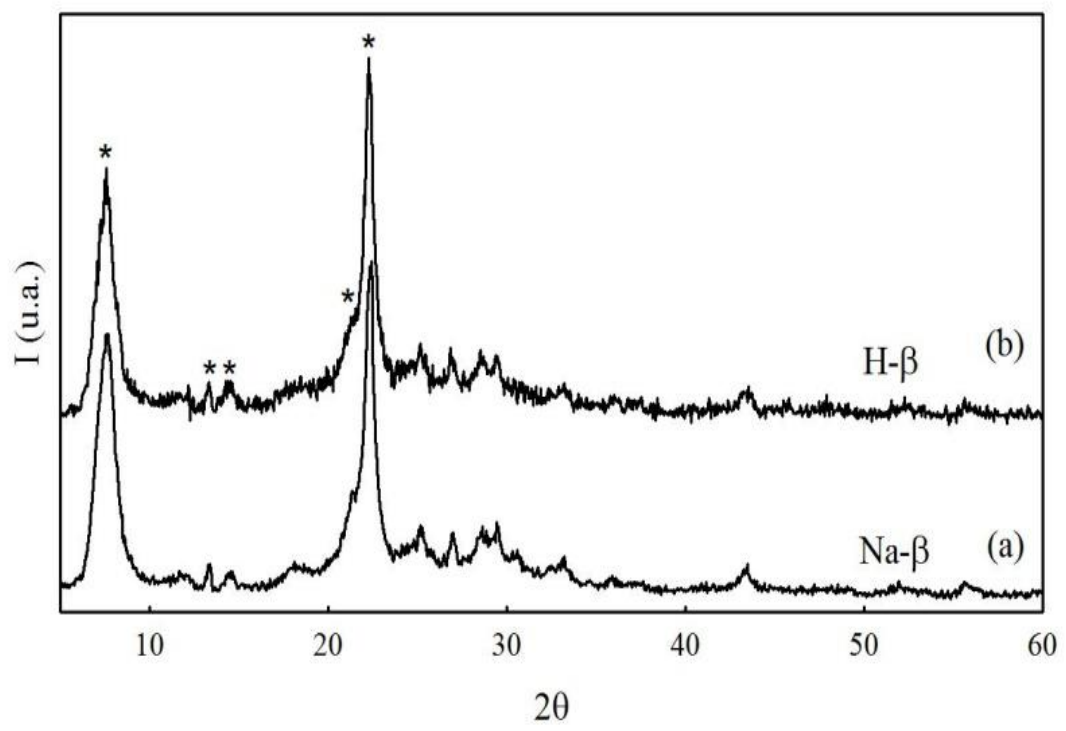

Figura 2 - Difratogramas de raios-X da zeólita beta, na forma (a) sódica e (b) ácida. 


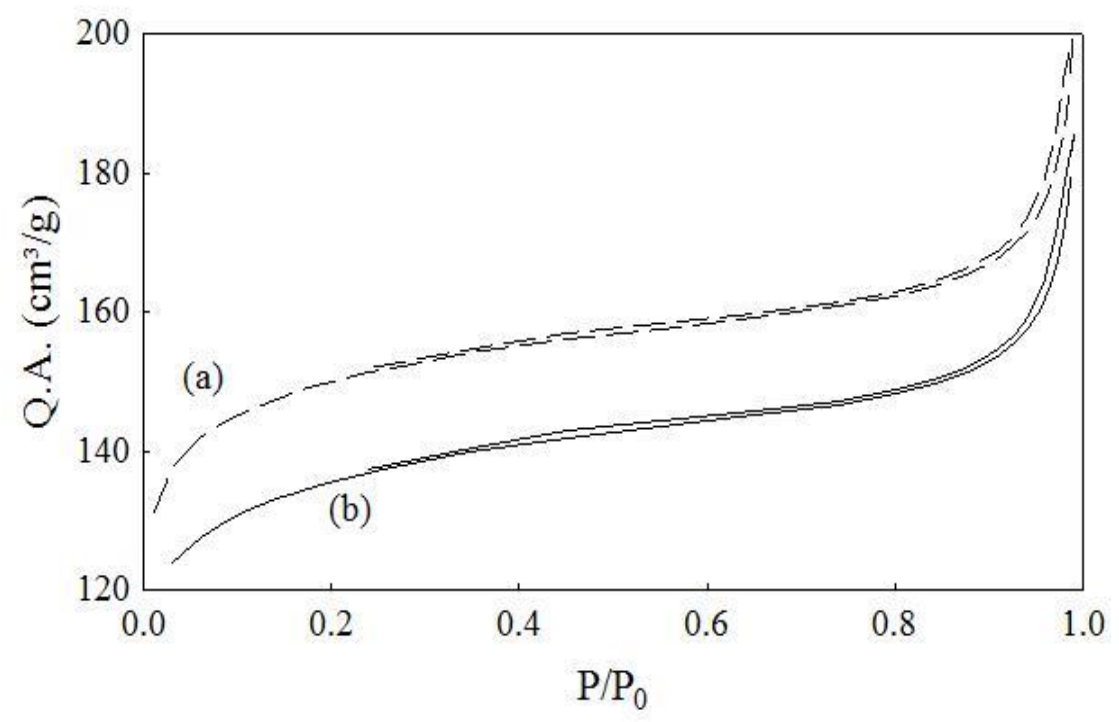

Figura 3 - Perfil de adsorção/dessorção de N2 a 77 K, para a zeólita beta, na forma (a) sódica e (b) ácida

poros. Enquanto, sua forma ácida apresentou área específica levemente inferior, $458 \mathrm{~m}^{2} / \mathrm{g}$, volume de poros também pouco menores, $0,277 \mathrm{~cm}^{3} / \mathrm{g}$, e tamanho médio de poros semelhante, $24 \AA$. As propriedades texturais são compatíveis com resultados apresentados na literatura (CORMA et al., 2003; XU et al., 2008) e não foram significativamente alteradas após a troca iônica, apresentando um pequeno decréscimo na área específica e volume de poros decorrente deste processo. Entretanto, a estrutura da zeólita ficou mantida praticamente inalterada.

Como a zeólita beta é classificada como uma zeólita de alto teor de sílica, a formação da estrutura cristalina está diretamente relacionada à reatividade no gel de síntese da fonte de silício utilizada como material precursor. Nesse caso, visto que a formação da estrutura zeolítica ocorreu após um curto tempo de cristalização, é possível afirmar que a metodologia empregada para extração da sílica de cinzas de casca de arroz permite obter sílica altamente reativa, podendo ser utilizada na síntese de diversos materiais.

As amostras de zeólita beta nas formas sódica e ácida foram testadas como catalisadores para a desidratação de isopropanol a propeno. Os produtos de reação foram analisados por cromatografia gasosa. A vazão molar de álcool na alimentação foi calculada considerando que, no saturador, o álcool líquido está em equilíbrio com o seu vapor usando a equação de Antoine (CENSKY et al., 2010). Para as condições experimentais empregadas, a vazão de isopropanol alimentado foi de $32 \mathrm{mmol} / \mathrm{h}$.

As vazões molares de cada componente na saída do reator, Fi, foram calculada a partir do balanço molar, Equação 1, admitido-se que a vazão molar de átomos de carbono é constante e que, pela estequiometria da reação, Equação 2, a vazão de água produzida é igual à de propeno. A fração molar de cada componente orgânico foi obtida pela análise cromatográfica. A conversão de isopropanol, Xisop, foi calculada a partir das vazões molares na entrada e na saída do reator, Equação 3.

$$
\begin{aligned}
& F_{\text {isop,entrada }}+F_{\mathrm{N}_{2}}=F_{\text {orgânicos }}+F_{\mathrm{H}_{2} \mathrm{O}}+F_{\mathrm{N}_{2}} \\
& \mathrm{H}_{3} \mathrm{C}-\mathrm{HCOH}-\mathrm{CH}_{3} \rightarrow \mathrm{H}_{2} \mathrm{C}=\mathrm{CH}-\mathrm{CH}_{3}+\mathrm{H}_{2} \mathrm{O} \\
& \text { Isopropanol } \quad \text { Aropeno } \quad \text { Aua } \\
& X_{\text {isop }}=\frac{\left(F_{\text {isop,entrada }}-F_{\text {isop,saida }}\right)}{F_{\text {isop,entrada }}}
\end{aligned}
$$

Na Figura 4, tem-se a conversão do isopropanol em função da temperatura de reação. Para a zeólita beta na forma sódica $(\mathrm{Na}-\beta)$, em temperaturas de reação abaixo de $150{ }^{\circ} \mathrm{C}$ não se obteve conversão significativa. Com o aumento da temperatura entre 150 e $200{ }^{\circ} \mathrm{C}$ houve um pequeno aumento na conversão. Contudo, foi entre $200 \mathrm{e}$ $225^{\circ} \mathrm{C}$ que a conversão passou de $12 \%$ para 74 $\%$, chegando a $100 \%$ na temperatura de reação de $250^{\circ} \mathrm{C}$. Por outro lado, com a forma ácida da zeólita beta (H- $\beta$ ), foi possível obter conversão de $100 \%$ de isopropanol em temperaturas bem inferiores, a partir de $150{ }^{\circ} \mathrm{C}$. O deslocamento da temperatura inicial de conversão completa de 


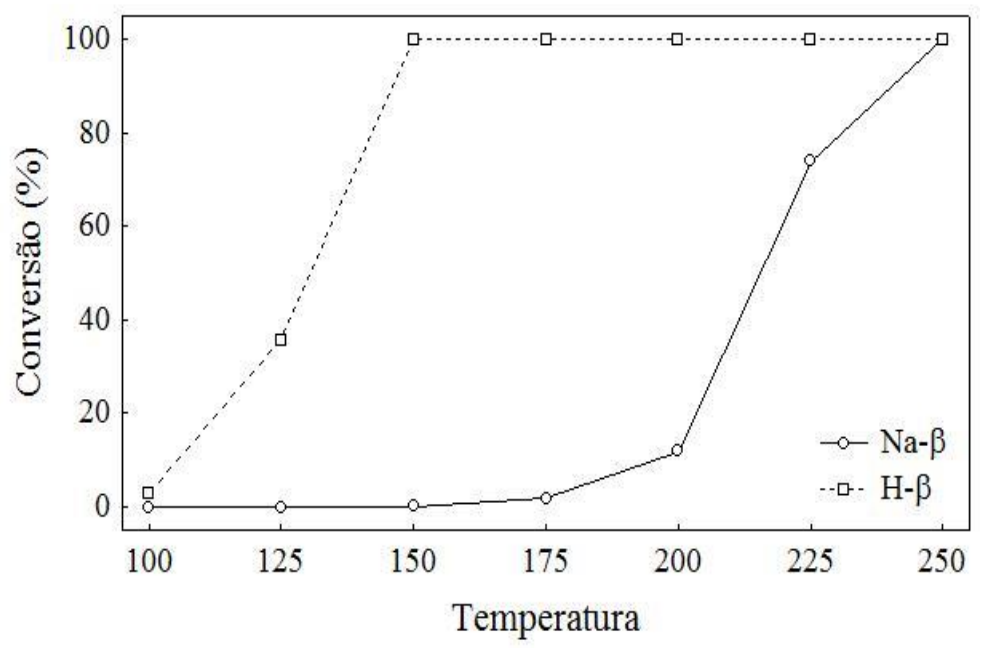

Figura 4 - Conversão de isopropanol sob zeólita beta em diferentes temperaturas.

isopropanol para temperaturas mais baixas com o catalisador na forma ácida, em comparação com sua forma sódica, indica que o processo de troca iônica aumentou a atividade catalítica da zeólita, ou seja, houve a troca efetiva dos cátions $\mathrm{Na}+$ pelos cátions $\mathrm{H}+$, gerando a forma ácida mais ativa para reações de desidratação (FERENS et al., 2012).

A seletividade dos catalisadores na reação de desidratação de isopropanol pode ser observada pela composição dos produtos de reação, Figura 5. Esta reação não é limitada termodinamicamente, apresentando conversões de equilíbrio próximas a $100 \%$ em temperaturas superiores a $100{ }^{\circ} \mathrm{C}$, sendo a concentração de equilíbrio de isopropanol praticamente nula, e o produto principal propeno, já que a concentração de equilíbrio de éter diisopropílico é inferior a $1 \%$. O principal produto de reação é o propeno; enquanto o éter diisopropílico, único subproduto formado, não ultrapassa $0,6 \%$ do isopropanol convertido, Figura 5a.

Como a conversão de isopropanol ocorre

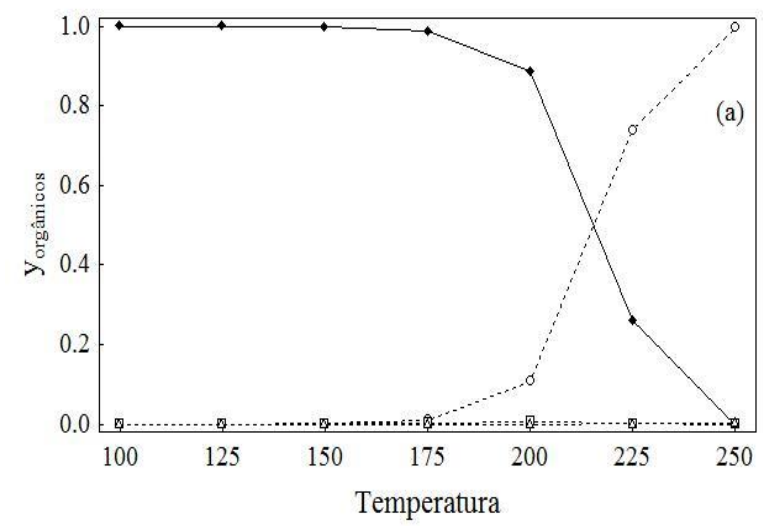

em temperaturas mais brandas para a zeólita na forma ácida, a fração de álcool decresce significativamente até $150^{\circ}$, a partir desta temperatura, seu valor é praticamente nulo. Enquanto o principal produto de reação continua sendo o propeno, com uma menor proporção de éter diisopropílico, nas temperaturas de reação superiores a $125^{\circ} \mathrm{C}$ houve pequena formação de outros subprodutos, Figura $5 \mathrm{~b}$, que aumenta com o aumento da temperatura, mas não excedendo $8 \%$ do total de reagente convertido.

Pode-se afirmar que os resultados obtidos com a zeólita beta sintetizada a partir de cinzas de casca de arroz são muito eficazes, visto que a conversão completa foi obtida a partir de $150^{\circ} \mathrm{C}$, com elevada seletividade para o propeno. Uma vez que ARAUJO e colaboradores (1999) obtiveram, para esta reação com zeólita SAPO-5, elevada seletividade a propeno e conversão máxima de isopropanol de cerca de $80 \%$, em temperaturas entre 150 e $340^{\circ} \mathrm{C}$. Enquanto, BEDIA e colaboradores

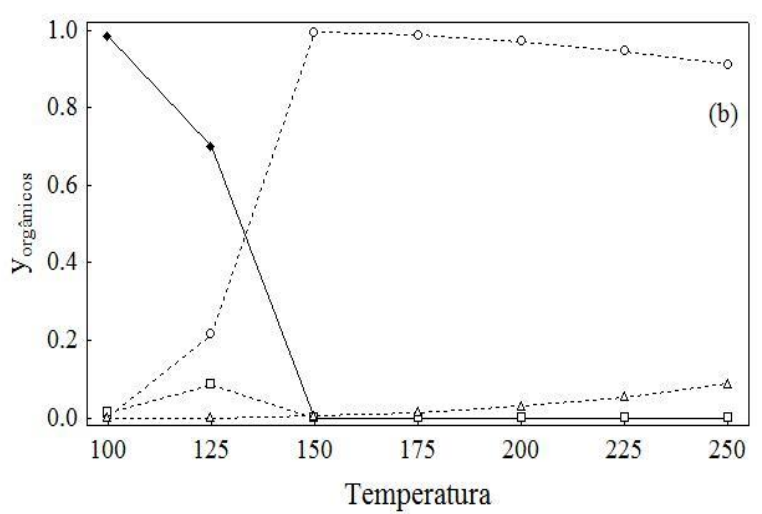

Figura 5 - Fração molar na fase orgânica dos produtos da conversão de isopropanol sob zeólita beta, na forma (a) sódica e (b) ácida. ( $\circ$ = propeno, $\bullet$ isopropanol; $\square=$ éter diisopropílico; $\Delta=$ outros subprodutos) 
(2010), utilizando carvão ativado impregnado com ácido fosfórico, obtiveram conversões de $100 \%$ a partir de $175^{\circ} \mathrm{C}$, com éter diisopropílico como único subproduto.

Não foi verificada a formação de acetona, produto da desidrogenação do isopropanol, sendo apenas observados propeno e éter diisopropílico, produtos de desidratação, além de compostos orgânicos com cadeias maiores, indicando apenas a presença de sítios ácidos nestas zeólitas. A conversão catalítica de isopropanol é considerada como uma reação modelo para a determinação da natureza de sítios ativos (ZURNACHYAN et al., 2011). A reação de desidratação ocorre em sítios ácidos, enquanto que a reação de desidrogenação formando acetona ocorre em sítios básicos (MANRÍQUEZ et al., 2004), que podem estar presentes também em catalisadores ácidos. A seletividade do catalisador para a reação de desidratação, em detrimento da reação de desidrogenação, permite afirmar que a zeólita beta apresenta elevada acidez, com atividade catalítica para reações catalisadas por ácidos.

Durante os testes reacionais houve mínima formação de subprodutos de oligomerização do propeno, mesmo em temperaturas de reação mais altas, para ambos catalisadores. Além disso, com a forma ácida da zeólita beta, a produção de propeno ocorre em temperaturas mais baixas. Estes catalisadores, sintetizados com sílica amorfa extraída de cinzas de casca de arroz, mostraram-se muito seletivos, podendo a reação ser realizada em condições reacionais brandas, demandando menor custo energético, produzindo propeno, insumo da indústria química e de polímeros, a partir do isopropanol, reagente que pode ser obtido a partir de matérias-primas renováveis.

\section{CONCLUSÕES}

Neste trabalho foi obtida sílica amorfa a partir de cinzas de casca de arroz, a qual foi utilizada com sucesso como material precursor para a síntese da zeólita beta, que apresentou estrutura cristalina bem definida e propriedades texturais com área específica elevada e mesoporos. A zeólita obtida apresentou atividade catalítica na reação de desidratação do isopropanol, tanto na forma ácida como na forma sódica. Observou-se seletividade de aproximadamente $100 \%$ para propeno e conversões de $100 \%$ em temperaturas de reação a partir de $150{ }^{\circ} \mathrm{C}$, mostrando a viabilidade da utilização de cinzas de casca de arroz como fonte de silício para a síntese de catalisadores com alta atividade e seletividade para a produção de propeno, um importante insumo da indústria petroquímica e de polímeros.

\section{AGRADECIMENTOS}

Os autores agradecem à Fundação de Amparo à Pesquisa do Estado do Rio Grande do Sul (FAPERGS) pela bolsa de estudo de Heveline Enzweiler.

\section{REFERÊNCIAS}

AN, D.; GUO, Y.; ZOU, B.; ZHU, Y.; WANG, Z. A green route to preparation of silica powders with rice husk ash and waste gas. Chemical Engineering Journal, v. 162, p. 509-514, 2010.

AN, D.; GUO, Y.; ZOU, B.; ZHU, Y.; WANG, Z. A study on the consecutive preparation of silica powders and active carbon from rice husk ash. Biomass and Bioenergy, v. 35, p. 1227-1234, 2011.

ARAUJO, A. S.; SOUZA, M. J. B.; FERNANDES Jr., V. J.; DINIZ, J. C. Kinetic study of isopropanol dehydration over silicoaluminiphosphate catalyst. React. Kinet. Catal. Lett., v. 66, p. 141-146, 1999.

BAJPAI, P. K.; RAO, M. S.; GOKHALE, K. V. G. K. Synthesis of mordenite type zeolite using silica from rice husk ash. Ind. Eng. Chem. Prod. Res. Dev., v. 20, p. 721-726, 1981.

BEDIA, J.; RUIZ-ROSAS, R.; RODRÍGUEZ-MIRASOL, J.; CORDERO, T. A kinetic study of 2-propanol dehydration on carbon acid catalysts. Journal of Catalysis, v. 271, p. 33-42, 2010.

CAMBLOR, M. A.; CORMA, A.; MARTÍNEZ, A.; MARTÍNEZ-SORIA, V.; VALENCIA, S. Mild Hydrocracking of Vacuum Gasoil over NiMo-Beta Zeolite Catalysts: The Role of the Location of the NiMo Phases and the Crystallite Size of the Zeolite. Journal of Catalysis, v. 179, p. 537-547, 1998.

CARVALHO, M. W. N. C.; CARDOSO, D. Synthesis of zeolite Beta with low template content. Studies in Surface Science and Catalysis, v. 105, p. 349-356, 1997.

CENSKY, M.; ROHAC, V.; RUZICKA, K.; FULEM, M.; AIM, K. Vapor pressure of selected aliphatic alcohols by ebulliometry. Part1. Fluid Phase Equilibria, v. 298, p. 192-198, 2010. 
CHANDRASEKHAR, S.; SATYANARAYANA, K. G.; PRAMADA, P. N.; RAGHAVAN, P.; GUPTA, T. N. Processing, properties and applications of reactive silica from rice husk - an overview. Journal of Materials Science, v. 38, p. 3159-3168, 2003.

CORMA, A. State of the art and future challenges of zeolites as catalysts. Journal of Catalysis, v. 216, p. 298-312, 2003.

CORMA, A.; DOMINE, M. E.; VALENCIA, S. Water-resistant solid Lewis acid catalysts: MeerweinPonndorf-Verley and Oppenauer reactions catalyzed by tin-beta zeolite. Journal of Catalysis, v. 215, p. 294-304, 2003.

CORMA, A.; GÓMEZ, V.; MARTÍNEZ, A. Zeolite beta as a catalyst for alkylation of isobutane with 2-butene: Influence of synthesis conditions and process variables. Applied Catalysis A: General, v. 119, p. 83-96, 1994.

DELLA, V. P.; KÜHN, I.; HOTZA, D. Rice husk ash as an alternate source for active silica production. Materials Letters, v. 57, p. 818-821, 2002.

DEY, K. P.; GHOSH, S.; NASKAR, M. K. Organic template-free synthesis of ZSM-5 zeolite particles using rice husk ash as silica source. Ceramics International, v. 39, p. 2153-2157, 2013.

DUAN, A.; WAN, G.; ZHANG, Y.; ZHAO, Z. JIANG, G.; LIU, J. Optimal synthesis of micro/ mesoporous beta zeolite from kaolin clay and catalytic performance for hydrodesulfurization of diesel. Catalysis Today, v. 175, p. 485-493, 2011.

FERENS, A. R.; WEINSTEIN, R. D.; GIULIANO, R.; HULL, J. A. Selective decomposition of isopropanol using as prepared and oxidized graphite nanofibers. Carbon, v. 50, p. 192-200, 2012.

JAMES, J.; RAO, M. S. Reaction product of lime and silica from rice husk ash. Cement and Concrete Research, v. 16, p. 67-73, 1986.

JANG, H. T.; PARK, Y. K.; KO, Y. S.; LEE, J. Y.; MARGANDAN, B. Highly siliceous MCM-48 from rice husk ash for $\mathrm{CO}_{2}$ adsorption. International Journal of Greenhouse Gas Control, v. 3, p. 545549, 2009.

KONG, L.; CHEN, H.; TAI, J.; SHEN, J.; ZHANG, S.; CHEN, J. Synthesis of small crystal zeolite beta in a biphasic $\mathrm{H}_{2} \mathrm{O}-\mathrm{CTAB}-$ alcohol system. Materials Letters, v. 63, p. 343-345, 2009.

KUMAR, N.; MÄKI-ARVELA, P.; YLÄSALMI, T.; VILLEGAS, J.; HEIKKILÄ, T.; LEINO, A. -R.; KORDÁS, K.; SALMI, T.; MURZIN, D. Y. Dimerization of 1-butene in liquid phase reaction: Influence of structure, pore size and acidity of Beta zeolite and MCM-41 mesoporous material. Microporous and Mesoporous Materials, v. 147, p. 127-134, 2012.

LARSEN, G.; LOTERO, E.; PETKOVIC, L. M.; SHOBE, D. S. Alcohol Dehydration Reactions over Tungstated Zirconia Catalysts. Journal of Catalysis, v. 169, p. 67-75, 1997.

MANRÍQUEZ, M. E.; LÓPEZ, T.; GÓMEZ, R.; NAVARRETE, J. Preparation of $\mathrm{TiO}_{2}-\mathrm{ZrO}_{2}$ mixed oxides with controlled acid-basic properties. Journal of Molecular Catalysis A: Chemical, v. 220, p. 229-237, 2004.

MARTÍNEZ, C.; CORMA, A. Inorganic molecular sieves: Preparation, modification and industrial application in catalytic processes. Coordination Chemistry Reviews, v. 255, p. 1558-1580, 2011.

MIKKELSEN, Ø.; KOLBOE, S. The conversion of methanol to hydrocarbons over zeolite H-beta. Microporous and Mesoporous Materials, v. 29, p. 173-184, 1999.

MYNSBRUGGE, J. V. der; VISUR, M.; OLSBYE, U.; BEATO, P.; BJØRGEN, M.; SPEYBROECK, V. V.; STIAN, S. Methylation of benzene by methanol: Single-site kinetics over H-ZSM-5 and H-beta zeolite catalysts. Journal of Catalysis, v. 292, p. 201-212, 2012.

O'CONNOR, C. T.; VAN STEEN, E.; DRY, M. E. New catalytic applications of zeolites for petrochemicals. Studies in Surface Science and Catalysis, v. 102, p. 323-362, 1996.

PRASETYOKO, D.; RAMLI, Z.; ENDUD, S.; HAMDAN, H.; SULIKOWSKI, B. Conversion of rice husk ash to zeolite beta. Waste Management, v. 26, p. 1173-1179, 2006.

RAMLI, Z.; LISTIORINI, E.; HAMDAN, H. Optimization and reactivity study of silica in the synthesis of zeolites from rice husk. Jurnal Teknologi, v. 25, p. 27-35, 1996. 
SANTI, D.; HOLL, T.; CALEMMA, V.; WEITKAMP, J. High-performance ring-opening catalysts based on iridium-containing zeolite Beta in the hydroconversion of decalin. Applied Catalysis A: General, v. 455, p. 46-57, 2013.

SARANGI, M.; NAYAK, P.; TIWARI, T. N. Effect of temperature on nano-crystalline silica and carbon composites obtained from rice-husk ash. Composites: Part B, v. 42, p. 1994-1998, 2011.

THUADAIJ, P.; NUNTIYA, A. Preparation and Characterization of Faujasite using Fly Ash and Amorphous Silica from Rice Husk Ash. Procedia Engineering, v. 32, p. 1026-1032, 2012.

TORRES, J. C.; CARDOSO, D. The influence of gel alkalinity in the synthesis and physicochemical properties of the zeolite [Ti,Al]-Beta. Microporous and Mesoporous Materials, v. 113, p. 204-211, 2008.

WANG, H. P.; LIN, K. S.; HUANG, Y. J.; LI, M. C.; TSAUR, L. K. Synthesis of zeolite ZSM-48 from rice husk ash. Journal of Hazardous Materials, v. 58, p. 147-152, 1998.

WANG, Y.; MIN, E.; MU, X. Nanosized $\beta$ zeolites and their composites as catalysts for acylation and alkane isomerization. Studies in Surface Science and Catalysis, v. 154, p. 241-249, 2004.

WEST, R. M.; BRADEN, D. J.; DUMESIC, J. A. Dehydration of butanol to butane over solid acid catalysts in high water environments. Journal of Catalysis, v. 262, p. 134-143, 2009.

XU, X.; ZHAO, X.; SUN, L.; LUI, X. Adsorption separation of carbon dioxide, methane, and nitrogen on $\mathrm{H} \beta$ and Na-exchanged $\beta$-zeolite. Journal of Natural Gas Chemistry, v. 17, p. 391-396, 2008.

YIN, C.; TIAN, D.; XU, M.; WEI, Y.; BAO, X.; CHEN, Y.; WANG, F. One-step synthesis of hierarchical mesoporous zeolite Beta microspheres from assembly of nanocrystals. Journal of Colloid and Interface Science, v. 397, p. 108-113, 2013.

ZAIKU, X.; QINGLING, C.; BO, C.; CHENGFANG, Z. Influence of alkalinity on particle size distribution and crystalline structure in synthesis of zeolite beta. Crystal Engineering, v. 4, p. 359-372, 2001.

ZHANG, H.; XIE, B.; MENG, X.; MÜLLER, U.;
YILMAZ, B.; FEYEN, M.; MAURER, S.; GIES, H.; TATSUMI, T.; BAO, X.; ZHANG, W.; VOS, D. D.; XIAO, F. -S. Rational synthesis of Beta zeolite with improved quality by decreasing crystallization temperature in organotemplate-free route. Microporous and Mesoporous Materials, v. 180, p. 123-129, 2013.

ZULFUGAROV, Z.G.; SULEIMANOV, A.S.; SAMEDOV, Ch.R. Synthesis of Gallosilicate and Alumogermanate Zeolites and Investigation of their Activity in the Reaction of Alcohol Dehydration. Studies in Surface Science and Catalysis, v. 18, p. 167-174, 1984.

ZURNACHYAN, A. R.; MANUKYAN, Kh. V.; KHARATYAN, S. L.; MATYSHAK, V. A.; MNATSAKANYAN, R. A. New Isopropanol Dehydration Catalyst Based on Tungsten Carbide Prepared by Modified Self-Propagating High-Temperature Synthesis. Kinetics and Catalysis, v. 52, p. 851-854, 2011. 